\title{
Early weight gain predicts treatment response in adolescents with anorexia nervosa enrolled in a family-based partial hospitalization program
}

\author{
Jessica L. Van Huysse PhD ${ }^{1}$ (c) ｜ Kellsey Smith BS ${ }^{2}$ | Kathleen A. Mammel MD \\ Natalie Prohaska MD ${ }^{1}$ | Renee D. Rienecke $\mathrm{PhD}^{1,3,4}$ ๑)
}

${ }^{1}$ Department of Psychiatry, University of Michigan, Ann Arbor, Michigan

${ }^{2}$ Department of Pediatrics, University of Michigan, Ann Arbor, Michigan

${ }^{3}$ Department of Pediatrics Medical, University of South Carolina, Charleston, South Carolina

${ }^{4}$ Department of Psychiatry and Behavioral Sciences, Medical University of South Carolina, Charleston, South Carolina

\section{Correspondence}

Jessica L. Van Huysse, Michigan Medicine Department of Psychiatry, 1500 East Medical Center Dr. F6303, SPC 5277, Ann Arbor, MI 48109.

Email: jvanhuy@med.umich.edu

\section{Present address}

Renee D. Rienecke PhD, Director of Research, Eating Recovery Center/Insight and Department of Psychiatry and Behavioral

Sciences, Northwestern University

Action Editor: Tracey Wade

\begin{abstract}
Objective: Improved treatment outcome in family-based treatment (FBT) for anorexia nervosa (AN) is predicted by weight gain occurring early in the course of treatment (i.e., about 4 lbs by week 4). Although prior work suggests that early weight gain in higher levels of care (e.g., partial hospitalization programs [PHP]) predicts weight restoration at discharge, no study has examined the specific rate of gain within FBT-informed PHP programs that best predicts treatment response.

Method: This study examined rate of weight gain in pounds and percent expected body weight (EBW) that predicts positive outcome in 70 patients $(M$ age $=15.49$ years, $\mathrm{SD}=2.56$ ) with $\mathrm{AN}$ who were enrolled in a family-based PHP.

Results: Receiver operator characteristic analyses demonstrated that changes in \% EBW during weeks 2-5 were more useful than changes in weight in predicting positive outcome. Gaining at least 8.9 pounds or over $8 \%$ of EBW in the first 4 weeks of treatment significantly predicted positive outcome.

Discussion: Findings suggest that positive outcome in an FBT-informed PHP is predicted by rapid weight gain in the initial weeks of treatment. Research is needed to identify specific family and patient characteristics that facilitate weight gain and to develop corresponding interventions to improve outcome.
\end{abstract}

\section{KEYWORDS}

adolescent, anorexia nervosa, body weight, treatment outcome, weight gain

\section{1 | INTRODUCTION}

Family-based treatment (FBT) for anorexia nervosa (AN) is a manualized outpatient treatment (Lock \& Le Grange, 2012) that is more effective than individual therapy in promoting weight restoration (Lock et al., 2010). Despite the efficacy of FBT, there are ongoing efforts to improve the intervention, given remission rates between 29 and 48\% at 12-month follow-up (Le Grange et al., 2016; Lock et al., 2010). Predictors of treatment response have been investigated, with the goal to identify modifiable predictors of treatment outcome (Le Grange et al., 2012). One of the most consistent predictors of remission in FBT is weight gain during the initial weeks of treatment, where gain of about $4-5$ pounds $(1.8-2.3 \mathrm{~kg})$, or $2.88 \%$ expected body weight (EBW), by session 3-4 of FBT, predicts remission at the end of FBT (Doyle, Le Grange, Loeb, Doyle, \& Crosby, 2010; Le Grange, Accurso, Lock, Agras, \& Bryson, 2014; Lock, Couturier, Bryson, \& Agras, 2006; Madden et al., 2015).

Given the utility of FBT, treatment programs utilizing higher levels of care (e.g., partial hospitalization programs [PHP]), have integrated FBT strategies into treatment protocols (Richards, Subar, Touyz, \& Rhodes, 2017). Although all aspects of manualized FBT cannot be translated to a PHP, family-based PHPs attempt to reproduce core FBT tenets 
(e.g., focusing on parental empowerment and discouraging reliance on the program for refeeding; see Hoste, 2015). Only one study has specifically investigated early weight gain in the context of a family-based PHP, utilizing logistic regression to demonstrate that \%EBW gained in the first 4 weeks of treatment is predictive of weight restoration at PHP discharge (Martin-Wagar, Holmes, \& Bhatnagar, 2018). The literature in non-familybased PHPs is similar, as higher rates of weight gain in the initial stages of treatment predict successful treatment outcomes (Bégin, GagnonGirouard, Aimé, \& Ratté, 2013; Makhzoumi et al., 2017; Nazar et al., 2017). Together, findings provide support for early weight gain as a predictor of outcomes in the PHP setting. However, prior work in PHPs has not investigated rates or timing of weight gain that best predicts outcomes. Given the intensive nature of PHPs, weight gain cut-points observed in manualized FBT may be insufficient for predicting treatment response in a PHP.

The current study aimed to extend prior findings by investigating the rate of weekly weight restoration that predicts positive outcome in patients with AN within a family-based PHP. We examined the amount of weekly weight gain that is predictive of positive outcome using both gain in pounds and increase in \%EBW. Because outcome was determined at the end of PHP, before expected remission of the eating disorder (ED), we defined a positive outcome as achieving $\geq 95 \%$ EBW and $\leq 2$ SDs of community norms on the Eating Disorder Examination (EDE).

\section{2 | METHODS}

\section{1 | Participants}

Participants $(n=70)$ in this observational study represented sequential admissions to a family-based PHP. Patients were between the ages of 10 and 19 ( $M$ age $=15.49$ years, SD $=2.56$ ) and met DSM-5 criteria for AN (see Supplementary Figure 1 for details on participant inclusion). The majority of patients were female (91.4\%), Caucasian (95.7\%), and from intact families (77.1\%). Diagnoses were made via chart review, which integrated information obtained from the initial medical and psychiatric evaluations and information obtained from the patient during the EDE, completed by program staff. Two raters (JV and other program staff) independently assigned the diagnoses with high (92\%) agreement on AN diagnosis. Kappa was unable to be calculated due to lack of variability in one rater's diagnosis in this sample. Discrepant diagnoses were resolved via chart review. Participants were required to meet all DSM-5 criteria for $A N$, with low weight defined as $\leq 89 \%$ median body mass index (BMI) for age and sex as in prior work (Sawyer, Whitelaw, Le Grange, Yeo, \& Hughes, 2016). Forty-five additional patients were excluded due to incomplete end of treatment EDE data. Excluded patients did not differ significantly from participants in baseline characteristics (\%mBMI, \% EBW, global EDE scores, or age). Patients were recruited to participate at the time of program entry, and this study represents secondary analyses of that dataset. Patients were informed that they may decline research participation with no impact on their treatment. The data used in this study are available from the corresponding author upon reasonable request. This study was approved by the IRB, and assent/consent was obtained from patients/parents.

Programming in the PHP is offered Monday-Friday for 6 hours per day. The PHP is family-based, emphasizing phase 1 of FBT (Lock \& Le Grange, 2012). Parental participation is required, including choosing all meals and snacks for their child, weekly family sessions with their PHP FBT therapist, and parent participation in group debriefing sessions and skills groups. As in phase 1 of FBT, the focus of the PHP is on parental management of food and other ED behaviors to facilitate weight restoration. Further details about this program are described elsewhere (e.g., Hoste, 2015; Smith, Van Huysse, \& Rienecke, in press).

\section{2 | Measures}

\subsection{1 $\quad$ Height, weight, and BMI}

BMI was calculated from weight and height taken by program staff. Measurements from the patient's first day of programming were used as baseline, and weight measurements 1 week later, 2 weeks later, and so on were used to assess cumulative weight and \%EBW changes. There were 10 cases who were missing weight data. To include these participants in analyses, an intent-to-treat strategy was utilized, incorporating the most recent observed weight value.

\subsection{2 | Eating-disorder examination}

The EDE (Cooper \& Fairburn, 1987) is a semi-structured interview designed to measure the cognitive and behavioral psychopathology of EDs. It consists of four subscales and a global score and has been found to have good psychometric properties (Berg, Peterson, Frazier, \& Crow, 2012). Internal consistency of the global score was excellent in this sample $(\alpha=0.92)$. Patients completed the baseline EDE at intake (64\%), the first day of PHP (33\%), or within the first week of treatment (3\%). Follow-up EDEs were completed at PHP discharge (within 1 week [61\%], 2 weeks [32\%], or 3 weeks [8\%] of discharge).

\subsubsection{Consensus EBW}

Consensus EBW ("EBW") was determined by the treatment team based on review of individual growth charts and other relevant information (e.g., pubertal development). Consensus EBW was set above the mBMI $40 \%$ of the time, due to individual growth histories above the 50 th percentile. On average, the consensus EBW versus $\mathrm{mBMI}$ differed by slightly under one $B M I$ point ( $M$ difference $=0.94, S D=1.04$ ). Although FBT clinical trials have defined EBW based on median BMI (e.g., Lock et al., 2010), there are prior observational studies that have used approaches similar to the consensus EBW (e.g., Martin-Wagar et al., 2018). 
TAB LE 1 Participant characteristics at baseline and end of PHP treatment

\begin{tabular}{|c|c|c|c|c|c|c|}
\hline \multirow[b]{2}{*}{ Characteristics } & \multicolumn{6}{|c|}{ Full sample $(\mathrm{N}=76)$} \\
\hline & $M(S D)$ & Range & $M(S D)$ & Range & $t(d f)$ & $p$-value \\
\hline$\% \mathrm{mBMI}$ & $80.0 \%(5.7 \%)$ & $64-89 \%$ & $91.9 \%(5.9 \%)$ & $78-108 \%$ & $-19.03(69)$ & $<0.01^{*}$ \\
\hline$\%$ Consensus EBW & $80.3 \%(5.8 \%)$ & $66-91 \%$ & $92.5 \%(5.0 \%)$ & $80-102 \%$ & $-19.94(69)$ & $<0.01^{*}$ \\
\hline PHP treatment duration (\# of treatment days) & $29.60(10.57)$ & $10-74$ & - & - & - & - \\
\hline
\end{tabular}

Abbreviations: $\mathrm{mBMI}$, median body mass index per CDC growth charts, based on age and sex; Consensus EBW, expected body weight as determined by treatment team; EOT, end of treatment; EDE, eating disorder examination; PHP, partial hospitalization program; M, mean; SD, standard deviation. Mean comparison presents results of paired samples $t$-test comparing baseline to end of PHP for relevant variables.

TAB LE 2 ROC analyses of weight gain predicting outcome at PHP completion

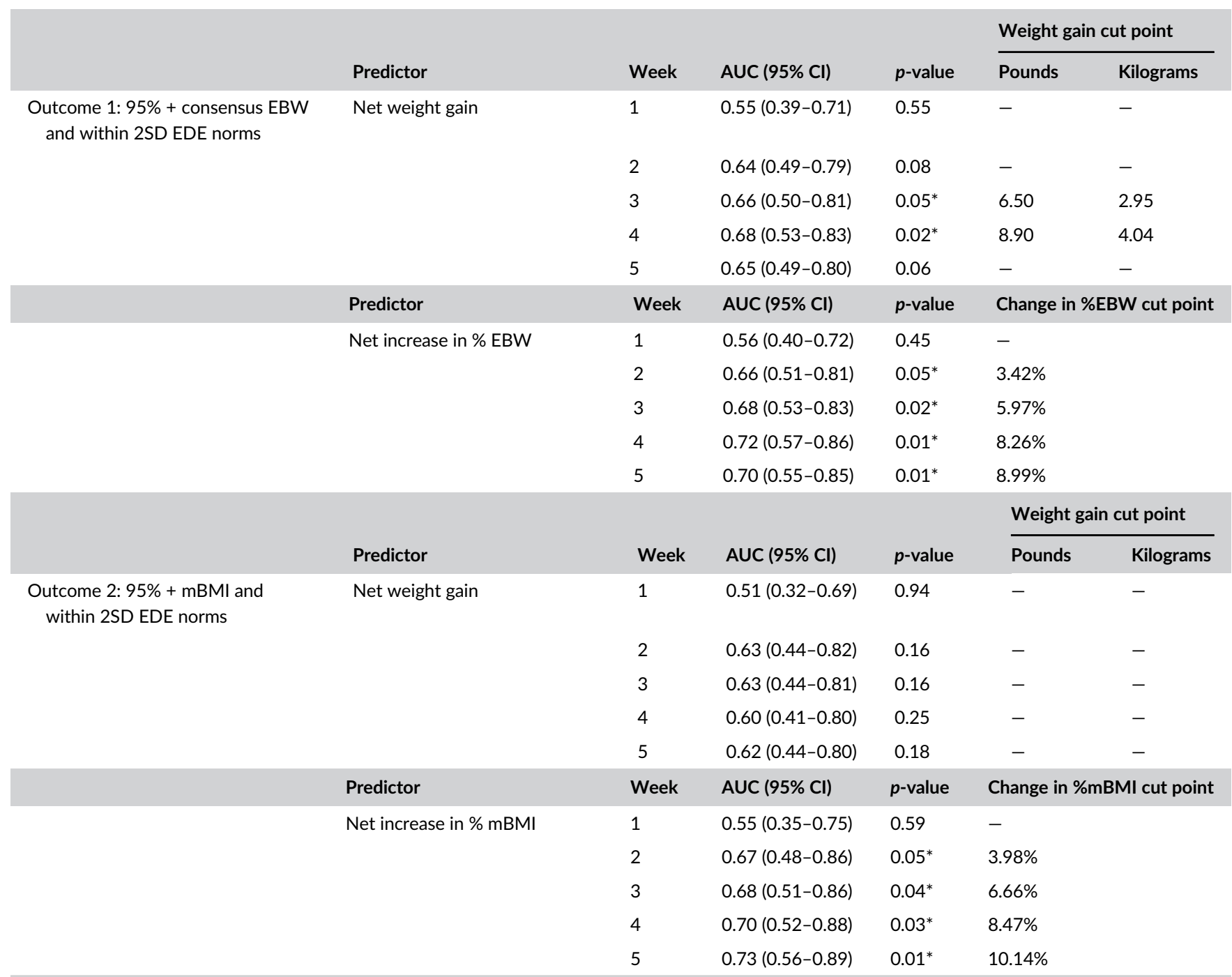

Abbreviations: ROC, receiver operating characteristic; PHP, Partial Hospitalization Program; AUC, area under the curve; consensus EBW, expected body weight as determined by treatment team; $\mathrm{mBMI}$, median body mass index per CDC growth charts for age and sex. Cut-points were determined based on the value that maximizes both sensitivity and specificity (i.e., occurring when sensitivity and specificity are about equal). Cut points are provided only for weeks that yielded statistically significant AUC values. Results for Outcome 1 and Outcome 2 are shown in the top and bottom halves of the table, respectively. ${ }^{*} p<0.05$. 


\subsection{4 $\mid$ Median BMI}

Median BMI for age and sex was determined based on CDC growth charts.

\section{3 | Statistical analyses}

Receiver operating characteristic (ROC) analyses were used to examine if early weight gain is predictive of treatment outcome, as well as the amount of weight gain that optimally predicts outcome. Cutpoints that achieved equal sensitivity and specificity were chosen. Weight gain during weeks 1-5 of treatment was examined, defined in separate analyses as gain in pounds (i.e., difference between weight during each week of treatment and baseline weight), gain in \%EBW (i.e., difference between \%EBW achieved and baseline \%EBW), and gain in \%mBMI (i.e., difference between \%mBMI achieved and baseline \%mBMI). Positive outcome was defined as obtaining a score within 2 SDs of community norms on the EDE and achieving $95 \%$ EBW (outcome 1), or the EDE criteria and 95\%mBMI (outcome 2). The ROC area under the curve (AUC) and corresponding 95\% confidence interval were used to evaluate the association between weight gain and treatment response.

\section{3 | RESULTS}

Patient characteristics and treatment outcomes are shown in Table 1. At the end of PHP, most participants reached 90\% EBW (78.6\%) or $90 \% \mathrm{mBMI}$ (62.9\%). Statistically significant increases in \% $\mathrm{mBMI}$ and \% EBW and decreases in EDE global scores, were observed (Table 1). Twenty-six percent of participants met the criteria of outcome 1 , whereas $19 \%$ met the criteria of outcome 2 .

Results of the ROC analyses are presented in Table 2. Weight gain in pounds only predicted outcome during weeks 3 and 4 , and only for outcome 1 . However, changes in both \%EBW and \%mBMI during weeks two through five significantly predicted positive outcome $(p<0.05)$. Most AUC values were in the 0.6-0.7 range, which can be considered similar to a moderate effect size (Rice \& Harris, 2005).

\section{4 | DISCUSSION}

This is the first study to investigate the rate of weight increase that predicts positive treatment outcome in a family-based PHP. Findings suggest that increase in \%EBW and \%mBMl during weeks 2 through 5 of enrollment in the PHP predicts positive treatment outcome in patients with AN and extends prior work (Martin-Wagar et al., 2018) by providing specific weight restoration thresholds that predict treatment response. Interestingly, weight gain in pounds was less predictive of outcome, with significant associations emerging only during weeks 3 and 4.
Overall, these findings suggest that rapid \%EBW/\%mBMI changes during a PHP program predict positive outcomes at discharge. Given differences in methodology, it is difficult to directly compare to similar studies in the manualized FBT literature, although it is notable that the \%mBMI cut points identified in the current study (e.g., $+8.5 \% \mathrm{mBMl}$ in first 4 weeks) suggest more rapid gain than in manualized FBT (e.g., +2.88\% EBW in first 4 weeks; Doyle et al., 2010). Follow-up data are needed to evaluate if weight gain during PHPs predicts longer term outcome. Research is also needed in other EDs that require weight restoration, as well as in more diverse samples.

The current study was based on an outcome definition that has been utilized in prior studies of FBT (Lock et al., 2006), yet is less stringent than some of the more recent trials which require scoring within one SD of EDE norms in addition to the weight criterion (Lock et al., 2010). We chose the less stringent criterion given that this study was limited to assessment of outcome at the conclusion of PHP, when full recovery is not yet expected. As described above, this definition resulted in $19-26 \%$ of participants being categorized as having a "positive outcome" at the end of PHP. Although this may appear low, we note the low starting \%EBW/mBMI $(80 \%$, see Table 1). Furthermore, we observed statistically significant improvements in \%EBW, \%mBMI, and EDE scores (see Table 1), similar to other FBT-informed and general PHP programs (see Friedman et al., 2016 for a review), and most participants achieved at least $90 \% \mathrm{mBM}$ before discharge.

Continued work is needed to clarify the best method to determine EBW in adolescents (Lebow, Sim, \& Accurso, 2018). Indeed, given the research-practice gap in terms of EBW definitions (i.e., research tends to utilize $\mathrm{mBMI}$, whereas clinicians tend to utilize individualized EBWs based on growth histories, Lebow et al., 2018), we conducted analyses using both definitions. The similar pattern of results across methods provides some support for utility of providerdetermined EBWs, although additional research is needed into the use of this strategy (e.g., inter-rater reliability of the provider EBW has not been investigated; Lebow et al., 2018).

Given the observational design of the current study, $39 \%$ of otherwise eligible patients were excluded due to missing EDE data (see Supplementary Figure 1). As noted above, excluded patients did not differ from included patients on key baseline characteristics. Given this, and the proportion of missing EDE data, complete case analyses were conducted (rather than imputation). Results should be interpreted with the rate of missing data in mind. Although specific reasons for missing EDEs are unknown, it may have been more likely in patients who had an unplanned discharge.

Taken together, this study adds to the literature demonstrating the importance of early weight gain in a family-based PHP in predicting outcomes and suggests specific cut-points for predicting positive response. It has been hypothesized that early weight gain is facilitated by caregivers who are especially effective at challenging the ED (Doyle et al., 2010). Improved understanding of these caregiver behaviors may inform therapeutic strategies that support rapid treatment response. 


\section{CONFLICT OF INTEREST}

Dr. Rienecke receives consulting fees from the Training Institute for Child and Adolescent Eating Disorders, LLC. Dr. Van Huysse, Ms. Smith, Dr. Mammel, and Dr. Prohaska report no conflicts of interest.

\section{DATA AVAILABILITY STATEMENT}

The data used in this study are available from the corresponding author upon reasonable request.

\section{ORCID}

Jessica L. Van Huysse (D) https://orcid.org/0000-0001-8179-7741

Renee D. Rienecke (D) https://orcid.org/0000-0003-0423-7403

\section{REFERENCES}

Bégin, C., Gagnon-Girouard, M.-P., Aimé, A., \& Ratté, C. (2013). Trajectories of eating and clinical symptoms over the course of a day hospital program for eating disorders. Eating Disorders: The Journal of Treatment and Prevention, 21(3), 249-264. https://doi.org/10.1080/10640266. 2013.779188

Berg, K. C., Peterson, C. B., Frazier, P., \& Crow, S. J. (2012). Psychometric evaluation of the eating disorder examination and eating disorder examination-questionnaire: A systematic review of the literature. The International Journal of Eating Disorders, 45, 428-438. https://doi.org/ 10.1002/eat.20931

Cooper, Z., \& Fairburn, C. (1987). The eating disorder examination: A semistructured interview for the assessment of the specific psychopathology of eating disorders. International Journal of Eating Disorders, 6, 1-8. https://doi.org/10.1002/1098-108X(198701)6:1<1::AIDEAT2260060102>3.0.CO;2-9

Doyle, P. M., Le Grange, D., Loeb, K., Doyle, A. C., \& Crosby, R. D. (2010). Early response to family-based treatment for adolescent anorexia nervosa. The International Journal of Eating Disorders, 43, 659-662. https://doi.org/10.1002/eat.20764

Friedman, K., Ramirez, A. L., Murray, S. B., Anderson, L. K., Cusack, A., Boutelle, K. N., \& Kaye, W. H. (2016). A narrative review of outcome studies for residential and partial hospital-based treatment of eating disorders. European Eating Disorders Review, 24, 263-276. https://doi. org/10.1002/erv.2449

Hoste, R. R. (2015). Incorporating family-based therapy principles into a partial hospitalization programme for adolescents with anorexia nervosa: Challenges and considerations. Journal of Family Therapy, 37, 41-60. https://doi.org/10.1111/1467-6427.12055

Le Grange, D., Accurso, E. C., Lock, J., Agras, S., \& Bryson, S. W. (2014). Early weight gain predicts outcome in two treatments for adolescent anorexia nervosa. The International Journal of Eating Disorders, 47, 124-129. https://doi.org/10.1002/eat.22221

Le Grange, D., Hughes, E. K., Court, A., Yeo, M., Crosby, R. D., \& Sawyer, S. M. (2016). Randomized clinical trial of parent-focused treatment and family-based treatment for adolescent anorexia nervosa. Journal of the American Academy of Child and Adolescent Psychiatry, 55, 683-692. https://doi.org/10.1016/j.jaac.2016.05.007

Le Grange, D., Lock, J., Agras, W. S., Moye, A., Bryson, S. W., Jo, B., \& Kraemer, H. C. (2012). Moderators and mediators of remission in family-based treatment and adolescent focused therapy for anorexia nervosa. Behaviour Research and Therapy, 50, 85-92. https://doi.org/ 10.1016/j.brat.2011.11.003

LeBow, J., Sim, L. A., \& Accurso, E. C. (2018). Is there clinical consensus in defining weight restoration for adolescents with anorexia nervosa? Eating Disorders: The Journal of Treatment and Prevention, 26, 270-277. https://doi.org/10.1080/10640266.2017.1388664
Lock, J., Couturier, J., Bryson, S., \& Agras, S. (2006). Predictors of dropout and remission in family therapy for adolescent anorexia nervosa in a randomized clinical trial. International Journal of Eating Disorders, 39 , 639-647. https://doi.org/10.1002/eat.20328

Lock, J., \& Le Grange, D. (2012). Treatment manual for anorexia nervosa: A family-based approach (2nd ed.). New York, NY: Guilford Press.

Lock, J., Le Grange, D., Agras, W. S., Moye, A., Bryson, S. W., \& Jo, B. (2010). Randomized clinical trial comparing family-based treatment with adolescent-focused individual therapy for adolescents with anorexia nervosa. Archives of General Psychiatry, 67, 1025-1032. https://doi.org/10.1001/archgenpsychiatry.2010.128

Madden, S., Miskovic-Wheatley, J., Wallis, A., Kohn, M., Hay, P., \& Touyz, S. (2015). Early weight gain in family-based treatment predicts greater weight gain and remission at the end of treatment and remission at 12-month follow-up in adolescent anorexia nervosa. International Journal of Eating Disorders, 48, 919-922. https://doi.org/10. 1002/eat.22414

Makhzoumi, S. H., Coughlin, J. W., Schreyer, C. C., Redgrave, G. W., Pitts, S. C., \& Guarda, A. S. (2017). Weight gain trajectories in hospitalbased treatment of anorexia nervosa. International Journal of Eating Disorders, 50(3), 266-274. https://doi.org/10.1002/eat.22679

Martin-Wagar, C. A., Holmes, S., \& Bhatnagar, K. A. C. (2018). Predictors of weight restoration in a day-treatment program that supports family-based treatment for adolescents with anorexia nervosa. Eating Disorders, 27, 400-417. https://doi.org/10.1080/10640266.2018. 1528085

Nazar, B. P., Gregor, L. K., Albano, G., Marchica, A., Coco, G. L., Cardi, V., \& Treasure, J. (2017). Early response to treatment in eating disorders: A systematic review and a diagnostic test accuracy metaanalysis. European Eating Disorders Review: The Journal of the Eating Disorders Association, 25(2), 67-79. https://doi.org/10.1002/erv. 2495

Rice, M. E., \& Harris, G. T. (2005). Comparing effect sizes in follow-up studies: ROC area, Cohen's $d$, and r. Law and Human Behavior, 29, 615-620. https://doi.org/10.1007/s10979-005-6832-7

Richards, I. L., Subar, A., Touyz, S., \& Rhodes, P. (2017). Augmentative approaches in family-based treatment for adolescents with restrictive eating disorders: A systematic review. European Eating Disorders Review, 26, 92-111. https://doi.org/10.1002/erv.2577

Sawyer, S. M., Whitelaw, M., Le Grange, D., Yeo, M., \& Hughes, E. K. (2016). Physical and psychological morbidity in adolescents with atypical anorexia nervosa. Pediatrics, 137, e2015-e4080. https://doi.org/ 10.1542/peds.2015-4080

Smith, K. N., Van Huysse, J. L., \& Rienecke, R. D. (in press). Changes in meal-related anxiety predict treatment outcomes in an intensive family-based treatment program for adolescents with anorexia nervosa. Eating Disorders: The Journal of Treatment and Prevention., 1-12. https://doi.org/10.1080/10640266.2019.1688008

\section{SUPPORTING INFORMATION}

Additional supporting information may be found online in the Supporting Information section at the end of this article.

How to cite this article: Van Huysse JL, Smith K, Mammel KA, Prohaska N, Rienecke RD. Early weight gain predicts treatment response in adolescents with anorexia nervosa enrolled in a family-based partial hospitalization program. Int J Eat Disord. 2020;53:606-610. https://doi.org/10.1002/eat. 23248 